\title{
Characterization of subacute and convalescent fibrotic burden in the remote myocardium after acute infarction provides strong and incremental prediction of changes in left and right functions and final infarct size, incremental to knowledge of the subacute infarct size
}

\author{
Damien Mandry ${ }^{1 *}$, Evan Appelbaum', Bobby Heydari ${ }^{1}$, Shuaib Abdullah'1, Sanjeev Francis ${ }^{2}$, Heidi S Lumish ${ }^{3}$, \\ Brian B Ghoshhajra ${ }^{3}$, Udo Hoffmann ${ }^{3}$, Henry Gewirtz², Ron Blankstein', Yucheng Chen', Jiazuo H Feng ${ }^{1}$, \\ Karl-Philipp Kienle ${ }^{1}$, Rob J van der Geest ${ }^{4}$, Elliott Antman', Michael Jerosch-Herold ${ }^{5}$, Raymond Kwong ${ }^{1}$ \\ From 15th Annual SCMR Scientific Sessions \\ Orlando, FL, USA. 2-5 February 2012
}

\section{Summary}

To test the hypothesis that fibrotic burden in remote myocardium quantified by CMR during early period of infarct healing is a strong determinant of the cardiac remodeling outcome.

\section{Background}

After acute myocardial infarction (AMI), co-existing myocardial stunning, ischemia, and architectural alteration may yield variable patterns of ventricular remodeling with potential long-term prognostic implications. We hypothesize that CMR quantification of fibrotic content, based on R1 (1/T1) assessment, in non-infarct myocardium early after infarction and during the infarct healing phases may provide novel prediction of the final infarct size and alteration of ventricular functions during infarct healing.

\section{Methods}

We quantified infarct size by late gadolinium enhancement (LGE), mean segmental fibrotic index $\left(\mathrm{FI}_{\text {MeanSeg }}\right)$ and total LV fibrotic burden ( $\left.\mathrm{FI}_{\text {Total }}\right)$, ventricular function and sizes during subacute (2-4 weeks) and chronic

${ }^{1}$ Cardiovascular Division, Department of Medicine, Brigham and Women's Hospital, Boston, MA, USA

Full list of author information is available at the end of the article stages (6 months) of healing in 62 AMI patients (45 men, mean LVEF $55 \pm 8 \%$ ). Infarct-healing period was defined as the time between the subacute and the chronic stages. For the purpose of quantifying $\mathrm{FI}_{\text {MeanSeg }}$ and $\mathrm{FI}_{\text {Total }}$, we serially quantified the ratio of myocardial to blood pool R1 changes (from before, and up to 30 minutes after contrast injection) to measure the expansion of the extracellular matrix as a marker of fibrosis. Three parallel short-axis slices were acquired for fibrotic quantification using a cine-IR sequence and slicematched LGE imaging. Myocardial segments with any LGE were excluded from quantification of fibrotic indices. Serum hematocrit was used to estimate the plasma R1 from the measured blood R1.

\section{Results}

From subacute to chronic stage, $\mathrm{FI}_{\text {MeanSeg }}$ in remote myocardium increased significantly (from $0.32 \pm 0.05$ to $0.35 \pm 0.07, \mathrm{P}=0.008$ ). Severity of increased $\mathrm{FI}_{\text {MeanSeg }}$ during the infarct-healing period was correlated to the severity in worsening of $\operatorname{LVEF}(\mathrm{r}=-0.27, \mathrm{P}=0.03)$, trend worsening of LVESV $(\mathrm{r}=0.24, \mathrm{P}=0.07)$, and significant worsening of RVESV ( $r=0.34, P=0.009)$. Linear regression demonstrated that $\mathrm{FI}_{\text {MeanSeg }}$ was one of the strongest predictors of final infarct mass (in grams) at the chronic stage. Specifically, both $\mathrm{FI}_{\text {MeanSeg }}$ at the subacute 
stage and its change during the infarct-healing period, demonstrated strong and independent association with the extent of the final infarct mass at the chronic stage, incremental to infarct mass measured at the subacute stage $(\mathrm{P}=0.004$ and $\mathrm{P}=0.02$, respectively, for improved model F-value).

\section{Conclusions}

Quantification of non-infarcted fibrotic burden during subacute stage and its change during infarct-healing provides strong prediction of the severity of the LV and $\mathrm{RV}$ post-MI remodeling and of final infarct size at 6 months.

\section{Funding}

National Heart Lung and Blood Institute, National Institutes of Health (RO1-HL091157).

Dr. Mandry's salary is supported by the Societe Francaise de Radiologie (France).

\section{Author details}

${ }^{1}$ Cardiovascular Division, Department of Medicine, Brigham and Women's Hospital, Boston, MA, USA. ${ }^{2}$ Cardiology Division, Massachusetts General Hospital, Boston, MA, USA. ${ }^{3}$ Department of Radiology, Massachusetts General Hospital, Boston, MA, USA. ${ }^{4}$ Leiden University Medical Center, Leiden, Netherlands, USA. 'Department of Radiology, Brigham and Women's Hospital, Boston, MA, USA.

Published: 1 February 2012

- Convenient online submission

- Thorough peer review

- No space constraints or color figure charges

- Immediate publication on acceptance

- Inclusion in PubMed, CAS, Scopus and Google Scholar

- Research which is freely available for redistribution 